\title{
Optical Measurement Of Polyhedral Volume Based On DSP
}

\author{
Jinrong Zhou, Wenming Huang,Nan Zheng \\ College of Physics and Information Engineering, Minnan Normal University, Zhangzhou, Fujian, 363000, China
}

Keywords:Signal processing;Volume measurement;Laser ranging;Cloud scanning

\begin{abstract}
In the object volume measurement experiment, measuring cup water measurement method is one of the commonly used methods. This article set the volume of a rectangular pyramid measurement as an example, combined with laser principles and the CCD sensor imaging technology, puts forward a kind of fast automatic measurement.The test system uses CCD sensing, using the principle of laser ranging and the disk integral method, and through the DSP main control circuit to achieve the target of the cloud point acquisition and coordinate calculation, to achieve the object volume measurement.
\end{abstract}

\section{Introduction}

In the field of digital and automated measurement, the traditional contact measurement has been unable to meet the needs of high-precision and high-speed measurement.Non-contact measurement method has the advantages of simple structure, high measurement precision and good real-time performance. It is widely used in the field of measurement ${ }^{[1,2]}$.In the field of non-contact imaging measurement, many research institutions at home and abroad have studied the measurement error of CCD auto focus adjustment, cloud spot imaging and so on, and put forward the corresponding optimization method ${ }^{[3-6]}$.In order to realize the volume non-contact measurement of polyhedron, the mathematical model of measurement is deduced by laser triangulation and disk integral method.The experimental test system uses the software and hardware combination method, using high-speed DSP signal processor for the control core design pyramid volume optical measurement system ${ }^{[7]}$.

\section{Measurement principle}

The volume of the polyhedron is measured by the combination of the direct laser triangulation principle and the disk integration method ${ }^{[1,3,5]}$.Pyramid volume measurement uses the direct laser triangulation principle.The parallel light from the laser diode projects vertically to the surface of the measured object, forming a light spot, the spot diffuse in the surface, and some of the scattered light imaging on CCD through the receiving lens, when measured the surface moves or surface shape changes, the location of the imaging light subsequently changes.According to integral principle,the polyhedral volume can be approximated by the disc integration method, shown in figure1.

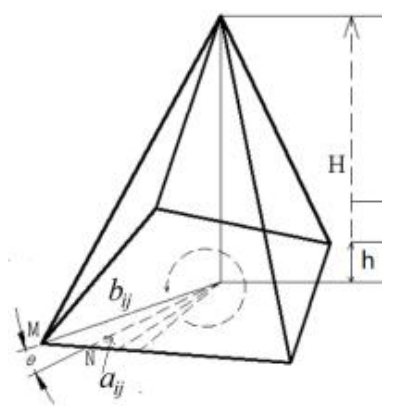

Fig.1 Disc integral measurement volume principle

In figure $1, \mathrm{By}$ the means of light on a point on the image sampling, using the method of laser triangulation to calculate the distance from sampling point of the corresponding object to the reference plane.After the semiconductor laser and CCD system scanning the surface of the under-tested object, it is easy to get a point cloud and the corresponding coordinate $P=(r, \theta, z)$,with $\mathrm{r}$ for cargo center of rotation to the point on the surface of the object distance, $\theta$ for cargo each rotation Angle, $z$ for rise high $h$.The center of the object is stage $O=(0,0,0)$.

Firstly, $a_{i j}$ and $b_{i j}$ is calculated the distance between the 
surface and center of rotation,using the disc integral measurement method.Then the wheel at the bottom is controlled to rotate $\theta$ each time, the point which is on the surface of the altimeter can be sampled, meanwhile the area of a triangle can be expressed as

$$
S_{i j}=\frac{1}{2} a_{i j} b_{i j} \sin \theta
$$

When the disc rotates one week, the area of the current layer can be calculated.After later, we can calculate the volume of the current layer by multiplying the bottom area by the height. Then we should elevate linear CCD and laser module for $h$ by the linear slide for the next measurement. Since then, the volume of the whole object can be expressed as

$V=\sum_{j=1}^{H / h} h_{j} \sum_{i=1}^{360^{\circ} / \theta} S_{i j}=\sum_{j=1}^{H / h}\left(h_{j} \times \sum_{i=1}^{360^{\circ} / \theta} \frac{1}{2} a_{i j} b_{i j} \sin \theta\right)$

If the last remaining distance of the top is less than $h$, because of the laser diffraction and scattering, part of the light will be CCD collected, therefore the problem of the lost at the top does not exist.

\section{Experimental Measurement Platform}

The experimental measurement platform is mainly composed of the DSP controller module with the core of TMS320F28335 high-speed processing, objective table rotation module, TSL1401 linear CCD, semiconductor laser source and LCD12864 LCD modules ${ }^{[8,9]}$. The system structure is shown in figure 2.

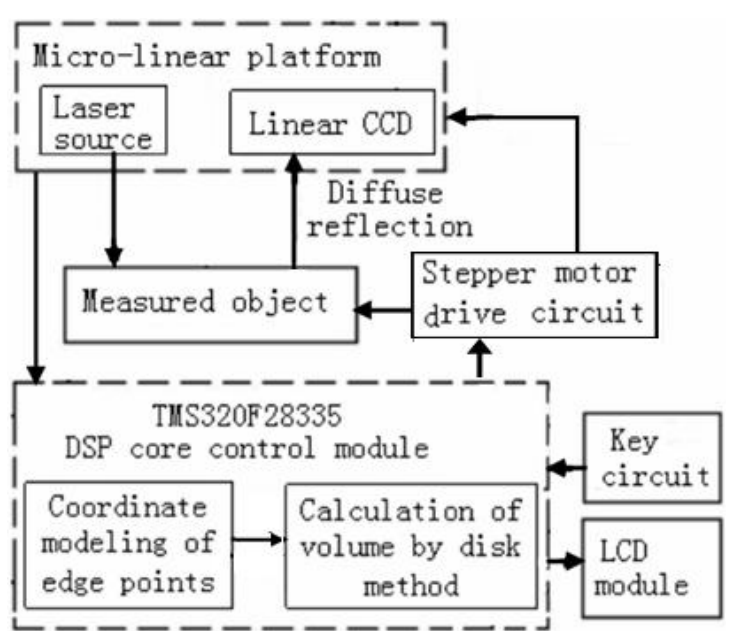

Fig. 2 System block diagram

\subsection{Master hardware circuit}

TMS320F28335 has rich resources of PWM pulse width modulation, powerful and fast floating-point data processing ability, which on the one hand controls the rotation of the stepper motor so as to drive the objective table rotation with the under-test object, rotate $10^{\circ}$ each time; on the other hand controls miniature linear slider (laser and linear CCD) to elevate step by step. When the semiconductor laser irradiates on the under-test object, the diffuse reflection produced by the objects are received by the linear CCD, linear CCD then upload data to TMS320F28335, the under-test object rises $1.2 \mathrm{~mm}$ then. When the TMS320F28335 receives the linear CCD acquisition of light point coordinate information, it will analyze and process, establishing the relevant mathematical model, and using the calculus in the disc method to calculate the volume of an object, shown the measurement results on LCD12864. The user can control the whole system through the button.

The objective table rotation and miniature linear slide unit module use L298N motor driver circuit to control the rotation of the objective table and the straight linear movement of the linear slipway. Stepper motor is the Open loop control elements which turn the electrical pulse signal into angular displacement or linear displacement. In the case of the non overload, motor speed, stop position only depend on the pulse signal frequency and pulse number, not the load change, which means that if a motor a pulse signal is given, a step from the Angle of motor is turning. This linear relations and the stepper motor with periodic error but cumulative error, made it easy to use stepper motor to control speed and position.

\section{2 software design}

For the software implementation, the TMS320F28335 chip of TI Company itself has A/D module which can collect the distance that CCD measure. For the collected data, we adopt the disc of calculus method to calculate the volume and complete the missing parts.For missing data, connects two recent point directly to form a complete triangle; Measurement results will be displayed on the LCD screen. The software controls the stepping motor to drive, and rotate. The main program flow chart are shown in figure 3. 


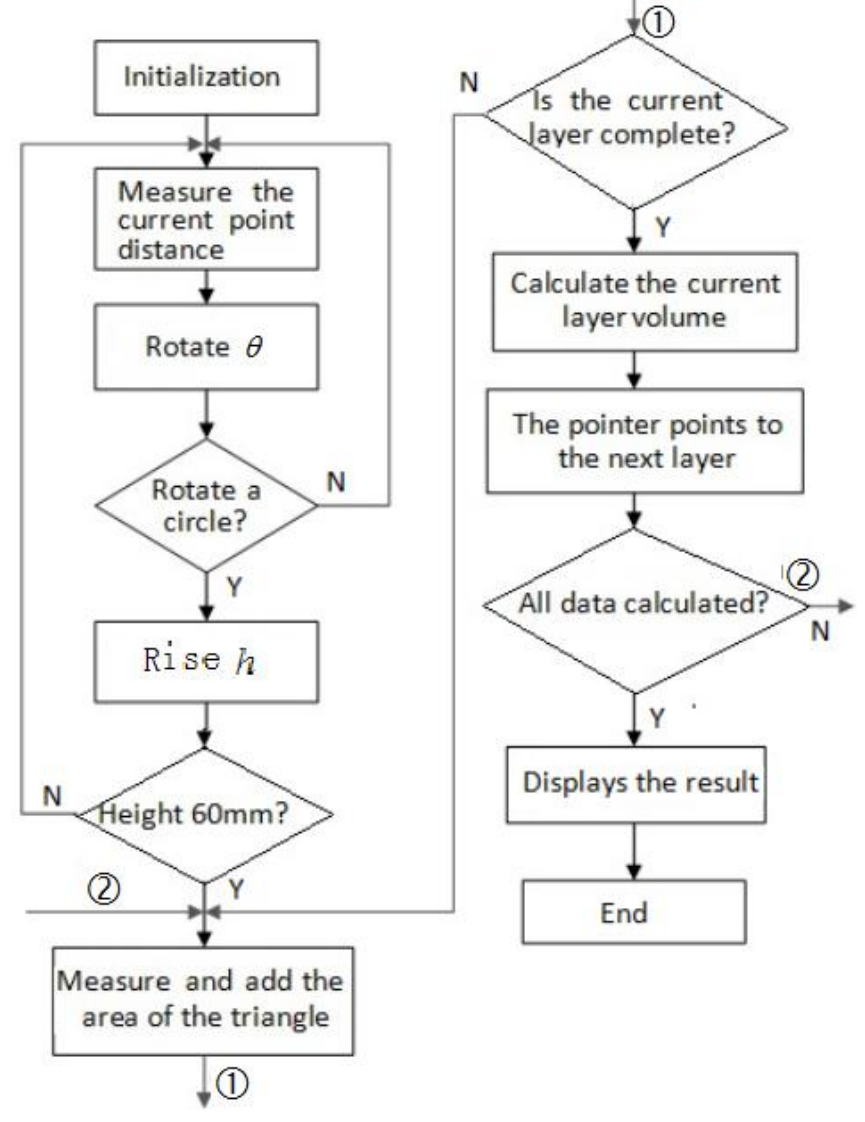

Fig.3 Main program flow chart

\section{System testing and results}

Before the experiment test,we should firstly implement the CCD correction test, making CCD curve fitting for the input testing data collected by EXCEL: Firstly,draw a coordinate diagram of the given data, determine its mathematics form through the chart.Secondly,confirm the undetermined parameters of the mathematical form.Finally, compare the actual measurement data and theory value after getting the mathematical model, measure the degree of judgment error, thus choosing appropriate parameters to minimized the mean square error.The volume of the pyramid for four different shape rubber blocks was measured by using the measuring cup drainage method and the design system. Assuming the polygonal volume measured by the measuring cup is the standard value.The Relative error between the value obtained by the laser measurement and the value measured by the measuring cup can be expressed as

$$
\delta=\frac{\Delta}{L} \times 100 \%
$$

The measurement results are shown in Table 1.

Tab. 1 Pyramid volume measurement results

\begin{tabular}{ccc}
\hline $\begin{array}{c}\text { Drainage method } \\
(\mathrm{ml})\end{array}$ & $\begin{array}{c}\text { Laser measurement } \\
\text { method }(\mathrm{ml})\end{array}$ & $\begin{array}{c}\text { Relative Error } \\
(\%)\end{array}$ \\
\hline 7.61 & 8.13 & 6.83 \\
13.42 & 12.56 & 6.41 \\
21.58 & 22.24 & 3.06 \\
35.45 & 33.98 & 4.15 \\
52.66 & 50.41 & 4.27 \\
\hline
\end{tabular}

\section{Conclusion}

In this design, adopting linear $\mathrm{CCD}$ sensor and using TMS320F28335 chip of TI Company, combined with laser three angle ranges, the least squares fitting and disc in calculus method to measure the volume of a pyramid, make the precision reach more than $90 \%$. Due to adopting high speed digital signal processing chip automatic measurement, the system has high measuring speed, less artificial dry and higher stability measurement.

\section{Acknowledgments}

This work is supported by Natural Science Foundation of Fujian Province,china(2016J01758), the Special Scientific Research Project of Fujian Province,china(JK2016026), the Science Project of Education Bureau of Fujian Province ,china(JAT160284).

\section{References}

[1] Wang Chen,Zhao Bin.Research of thin plate thickness measurement based on single lens laser triangulation[J].Laser Technology, 2013,37(1):6-9.

[2] Xing Jichuan,Luo Xiaohong.Measurement of truck carriage volume with laser triangulation[J]. Infrared and Laser Engineering,2012,41(11):3083-3087.

[3]Shen Lei,Li Dinggen,Chu Jun,et al.Study on laser speckle correlation method applied in triangulation displacement measurement $[\mathrm{J}]$. Infrared and Laser Engineering, 2014,43(1):288-293.

[4] Liu Jun,Liu Jiangchun,Yan Keun.Design of high precision automatic focusing system Based on Method of 
Optic Triangle[J].Journal of Xi'an University of laser triangulation displacement sensor system based on Technology,2004,20(1):63-66.

FPGA and DSP[J].Computer Measurement \&

[5] He Kai,Chen Xingwang,Jian Xin,et al.Analysis of error Control,2012,20(6):1725-1728.

in high precision laser triangulation displacement [8] Zhao Wanxin,Chen Siyi.Small intelligent patrol line measurement system[J]. Optics \& Optoelectronic Technology,2013,11(3):62-66. vehicle based on TSL1401 linear CCD[J].Industrial Control Computer, 2014,27(2 ):121-122.

[6] Li Yaqian,Fu Xianbin,Zhou Kun.Optical displacement

[9] Li Mengtao. Upright intelligent car design based on measuring system by CCD segmental measurement[J] . linear TSL1401 CCD $[\mathrm{J}]$. Electronic Design Engineering, Optics and Precision Engineering, 2011,19 (9) :2036-2042. 2013,21(21):86-88.

[7] Nie Chenchen,Zhang Haibo,Tao Wei,et al. Integrative 